\title{
Skipping breakfast is associated with lifestyle habits among Japanese pupils
}

\section{To the editor}

Breakfast is generally accepted to be the most important meal of the day, and it is known to impact health, well-being and cognitive performance. ${ }^{1)}$ Breakfast is also associated with sleep, and those who miss breakfast are reported to be poor sleepers. ${ }^{2)}$ No consistent conclusion has been reported on the association between breakfast and physical activity, ${ }^{3)}$ while lower physical activity in addition to higher screen time was reported to be significant predictors of skipping breakfast in Polish teenagers aged 11-13 years. ${ }^{4)}$ According to the same survey, in comparison to 'never-skippers,' 'frequent breakfast skippers' were more likely to be overweight/obese. Skipping breakfast was also associated with total and abdominal obesity in adolescents independent of sleep duration. ${ }^{5)}$ However, several reviews have reported that evidence on the role of breakfast frequency in adiposity outcomes across life span remains inconclusive. $\left.{ }^{6}\right)$ The importance of breakfast has also been supported by basic studies. For example, a genome-wide association study identified 6 genetic variants for breakfast skipping, linking clock regulation with food timing and suggesting a possible beneficial role of regular breakfast intake as part of a healthy lifestyle. ${ }^{7}$ However, the prevalence of skipping breakfast was reported to range from $10 \%-30 \%$, with an increasing trend in adolescents. ${ }^{8}$ The current cross-sectional study aimed to determine the association of skipping breakfast with various lifestyle habits in addition to academic performance and body mass index (BMI) among pupils in Japan.

The current study was part of a survey conducted between October 2016 and November 2018 to determine mutual associations among lifestyle habits and to clarify associations of lifestyle habits with pupils' health and academic performance. Details of the survey have been described elsewhere. ${ }^{9)}$ Of the 4,208 questionnaires collected from 28 public schools (15 elementary schools [ES], 8 junior high schools [JHS], and 5 high schools [HS]), 2,722 agreed to participate in the study and answered all the required questions. The selected numbers in the query (Supplementary Table 1) on breakfast, sleepiness, defecation, school day screen time, nonschool day screen time, and self-reported academic performance are termed as the breakfast score, sleepiness score, defecation score, school day screen time score, nonschool day screen time score, and self-reported academic performance score, respectively. Hours of after-school activity per week obtained by the product of the 2 numbers of the 2 queries (one on the frequency and the other on the duration), and the number of days engaged in physical activity per week was termed as after-school activity score and physical activity score, respectively. For dinner regularity, the choices 1-7 were categorized as regular dinner (dinner regularity score of 1 ) and choice 8 as irregular dinner (dinner regularity score of 2 ). The night-time sleep duration both before school days and nonschool days were calculated from bed and wake times.

Lifestyle habits associated with breakfast skippers were analyzed using a multivariable logistic regression model. Since frequency of eating breakfast was defined as follows (BF1, always; BF2, often; BF3, sometimes; BF4, never), breakfast skippers were defined as those with BF2, BF3, and BF4. ${ }^{4}$ Grade, gender (male was numbered as 1 and female as 2), sleepiness score, defecation score, screen time scores, self-reported academic performance score, after-school activity score, physical activity score, dinner irregularity score, the night-time sleep duration before school days, the night-time sleep duration before nonschool days, and standardized BMI were covariate factors adjusted on the multivariable logistic regression model. This study was approved by the Committee for Medical Research Ethics of Tokyo Bay Urayasu Ichikawa Medical Centre (No. 199).

In all school types, BF1 (ES, 90.9\%; JHS, 83.5\%; HS, 78.2\%) showed the highest rate, and the rate decreased to BF4 (ES, 0\%; JHS, 1.7\%; HS, 2.9\%) via BF2 (ES, 7.5\%; JHS, 11.9\%; HS, $12.1 \%$ ) and BF3 (ES, 1.6\%; JHS, 2.9\%; HS, 6.7\%). In contrast to the previous study, ${ }^{8)}$ the rate of BF4 pupils (who never took breakfast) was low in the current study. This may be partly due to the broad and longstanding campaign for taking breakfast supported by both the Ministry of Health, Labour and Welfare, and the Ministry of Agriculture, Forestry and Fisheries in Japan.

Although the current cross-sectional design study lacked data on demographic factors and age information, sleepiness (adjusted odds ratio [OR], 1.25; 95\% confidence interval [CI], $1.09-1.44 ; P=0.001)$, physical activity (OR, $0.91 ; 95 \% \mathrm{CI}$, $0.87-0.95 ; P<0.001)$, dinner irregularity (OR, $1.28 ; 95 \% \mathrm{CI}$, $1.01-1.62 ; P=0.039)$, school day screen time (OR, $1.34 ; 95 \%$ CI, 1.11-1.61; $P=0.002)$, academic performance (OR, 1.70; 95\% CI, 1.48-1.96; $P<0.001)$, after-school activity (OR, 1.03; 95\% CI, 1.01-1.04; $P<0.001)$, and sleep duration before school day (OR, 0.90; CL, 0.82-1.00; $P=0.040$ ) were independently associated with breakfast skippers (Table 1).

An association of skipping breakfast and poor academic performance is widely recognized, ${ }^{1)}$ and the present study supported 
Table 1. Multivariate logistic regression analysis of breakfast skippers versus never-skippers

\begin{tabular}{lccrrr}
\hline Variable & Partial regression coefficient (B) & SE & Wald $\chi^{2}$ & OR (95\% Cl) & $P$ value \\
\hline Sex & -0.17 & 0.12 & 2.03 & $0.85(0.67-1.06)$ & 0.154 \\
Sleepiness score & 0.23 & 0.07 & 10.22 & $1.25(1.09-1.44)$ & 0.001 \\
Physical activity score & -0.10 & 0.02 & 21.40 & $0.91(0.87-0.95)$ & $<0.001$ \\
Dinner irregularity score & 0.25 & 0.12 & 4.28 & $1.28(1.01-1.62)$ & 0.039 \\
School day screen time score & 0.29 & 0.09 & 9.68 & $1.34(1.11-1.61)$ & $\mathbf{0} .002$ \\
Nonschool day screen time score & 0.14 & 0.07 & 3.71 & $1.15(1.00-1.37)$ & 0.054 \\
Academic performance score & 0.53 & 0.07 & 55.04 & $1.70(1.48-1.96)$ & $<0.001$ \\
After-school activity score & 0.03 & 0.01 & 14.69 & $1.03(1.01-1.04)$ & $<0.001$ \\
Sleep duration before school days & -0.10 & 0.05 & 4.24 & $0.90(0.82-1.00)$ & $\mathbf{0 . 0 4 0}$ \\
\hline
\end{tabular}

$\mathrm{SE}$, standard error; OR, adjusted odds ratio; $\mathrm{Cl}$, confidence interval.

Covariates included in the logistic regression models: grade, sex, sleepiness score, physical activity score, dinner irregularity score, school day screen time score, nonschool day screen time score, academic performance score, defecation score, after-school activity score, standardized body mass index, sleep duration before school day, and sleep duration before nonschool day.

The presented model was obtained using a stepwise procedure. Its Akaike information criterion (2090.75) was lower than that obtained by all factors (2097.59) or that obtained by significant factors (2092.63).

Boldface indicates a statistically significant difference with $P<0.05$.

this association. The current finding on the association between skipping breakfast and sleep loss, though only on the night before school days, has also been reported, ${ }^{2)}$ and sleepiness was found to be associated with breakfast skipping. As previously demonstrated ${ }^{4)}$ an association between skipping breakfast and screen time, although only on school days, has been obtained. Regarding physical activity, similar to the study of Wadolowska et al. ${ }^{4}$ but in contrast to the study of Lyerly et al., ${ }^{3)}$ a lower physical activity score was found to be a significant predictor of an increase in skipping breakfast. Consistent with Hassan et al., ${ }^{6}$ the present study failed to demonstrate an association between skipping breakfast and higher BMI.

The current study might be the first study to show significant associations between skipping breakfast and an increase in afterschool activity as well as irregular dinner habits. In both Japan and Korea, after-school institutional extracurricular study was reported to be routine in order to prepare for qualification or entrance examinations among pupils. ${ }^{10)}$ Although longitudinal research should be conducted for confirmation, reducing screen time on school days, after-school activity, and irregular dinner habits is expected to decrease skipping breakfast, resulting in a decrease in pupils' sleepiness and an improved academic performance.

\section{Key message}

Question: Breakfast is important; however, adolescents are increasingly skipping it.

Finding: Skipping breakfast is independently associated with sleepiness, physical inactivity, dinner irregularity, screen time on school days, poor academic performance, after-school activity, and decreased sleep duration before school day.

Meaning: Reducing screen time on school days, after-school activity, and irregular dinner habits might decrease breakfast skipping, resulting in decreased sleepiness and improved academic performance.
Jun Kohyama, MD, PhD

Department of Paediatrics, Tokyo Bay Urayasu Ichikawa Medical Centre, Urayasu, Japan

Corresponding author: Jun Kohyama, MD, PhD, Department of Paediatrics, Tokyo Bay Urayasu Ichikawa Medical Centre, Toudaijima 3-4-32, Urayasu 279-0001, Japan

\ Email: j-kohyama@jadecom.or.jp, https://orcid.org/00000001-9793-7788

\section{Footnotes}

Conflicts of interest: No potential conflict of interest relevant to this article was reported.

Supplementary material: Supplementary Table 1 can be found via https://doi.org/10.3345/cep.2020.01263.

\section{References}

1. Rampersaud GC, Pereira MA, Girard BL, Adams J, Metzl JD. Breakfast habits, nutritional status, body weight, and academic performance in children and adolescents. J Am Diet Assoc 2005;105:743-62.

2. Agostini A, Lushington K, Kohler M, Dorrian J. Associations between selfreported sleep measures and dietary behaviours in a large sample of $\mathrm{Au}$ stralian school students $(\mathrm{n}=28,010)$. J Sleep Res 2018;27:e12682.

3. Lyerly JE, Huber LR, Warren-Findlow J, Racine EF, Dmochowski J. Is breakfast skipping associated with physical activity among U.S. adolescents? A cross-sectional study of adolescents aged 12-19 years, National Health and Nutrition Examination Survey (NHANES). Public Health Nutr 2014;17:896-905.

4. Wadolowska L, Hamulka J, Kowalkowska J, Ulewicz N, Gornicka M, Jeruszka-Bielaket M, et al. Skipping breakfast and a meal at school: its correlates in adiposity context. Report from the $\mathrm{ABC}$ of Healthy Eating Study of Polish Teenagers. Nutrients 2019;11:1563.

5. Forkert ECO, Moraes ACF, Carvalho HB, Manios Y, Widhalm K, 
González-Gross M, et al. Skipping breakfast is associated with adiposity markers especially when sleep time is adequate in adolescents. Sci Rep 2019;9:6380.

6. Hassan BK, Cunha DB, da Veiga GV, Pereira RA, Hoffman DJ, Sichieri R. Breakfast consumption, family breakfast, and adiposity trajectory in adolescence: The Adolescent Nutritional Assessment Longitudinal Cohort Study. J Acad Nutr Diet 2019;119:944-56.

7. Dashti HS, Jones SE, Wood AR, Lane JM, van Hees VT, Wang H, et al. Genome-wide association study identifies genetic loci for self-reported habitual sleep duration supported by accelerometer-derived estimates. Nat Commun 2019;10:1100.

8. Monzani A, Ricotti R, Caputo M, Solito A, Archero F, Bellone S, et al. A systematic review of the association of skipping breakfast with weight and cardiometabolic risk factors in children and adolescents: what should we better investigate in the future? Nutrients 2019;11:387.

9. Kohyama J, Ono M, Anzai Y, Kishino A, Tamanuki K, Moriyama K, et al. Factors associated with sleep duration among pupils. Pediatr Int 2020;62: 716-24.

10. Lee EY, Kang B, Yang Y, Yang HK, Kim HS, Lim SY, et al. Study time after school and habitual eating are associated with risk for obesity among overweight Korean children: a prospective study. Obes Facts 2018;11:46-55.

How to cite this article: Kohyama J. Skipping breakfast is associated with lifestyle habits among Japanese pupils. Clin Exp Pediatr 2021;64:433-5. https://doi.org/10.3345/cep.2020. 01263 